\title{
Bebida alcohólica por fermentación de cáscara y mucilago del café (Coffea arabica L.) a diferente $\mathrm{pH}$ y concentración de levadura
}

\section{Alcoholic beverage by fermentation of coffee husk and mucilage (Coffea arabica L.) at different $\mathrm{pH}$ and concentration of yeast}

\author{
Segundo Víctor Olivares Muñoz ; Meregildo Silva Ramírez²; Rosa Amalia Caruajulca Yoplac ${ }^{3}$
}

\section{RESUMEN}

La investigación tuvo por objetivo determinar el $\mathrm{pH}$ y porcentaje de levadura en la fermentación de cáscara y mucílago de café que permitan la elaboración de una bebida alcohólica con características fisicoquímicas y sensoriales para el consumo, para ello se estableció un diseño bifactorial 32 , el factor A el $\mathrm{pH}(3.5 ; 4 ; 4.5)$ y factor B el porcentaje de levadura $(0.5 \%, 1 \%, 1.5 \%)$; en la experiencia se evaluó el café, se obtuvo la cáscara y mucilago diluido en el pulpeado, se regulo el $\mathrm{pH}$ y adicionó la Saccharomyces cerevisiae para la fermentación a $20^{\circ} \mathrm{C}$, en un biorreactores de 10 L; se efectúo la evaluación fisicoquímica de acuerdo al análisis de varianza y comparaciones múltiples de Tuckey $(\alpha=0.05)$ y sensorial según la prueba de Friedman; se determinó que existe diferencia significativa entre tratamientos; sin embargo entre bloques (Tiempo de evaluación), no existió diferencia significativa. Se determinó que a pH 4.5 y adición de levadura seca comercial en 3\% en la fermentación de pulpa y cáscara de café permiten la elaboración de una bebida alcohólica con calificación sensorial entre bueno y muy bueno, con valores de color 8.15, aroma 7.82, aspecto 5.87 y sabor 7.24; asimismo densidad 1.029, $\mathrm{pH} 4.75$, solidos totales $2{ }^{\circ}$ Brix, acidez titulable $8.4 \mathrm{~g} / \mathrm{L}$, grado alcohólico $15.6 \mathrm{GL}$, extracto etéreo 0,193\%, proteína total $0.42 \%$, extracto libre de nitrógeno $0,692 \%$ y energía bruta $6.911 \mathrm{kCal} / 100 \mathrm{~mL}$.

Palabras clave: Levadura, evaluación, fermentación, fisicoquímico, sensorial.

\begin{abstract}
The objective of the research was to determine the $\mathrm{pH}$ and percentage of yeast in the fermentation of coffee husk and mucilage that allow the elaboration of an alcoholic beverage with physicochemical and sensory characteristics for consumption, for which a bifactorial design 32 was established, factor A the $\mathrm{pH}(3.5 ; 4 ; 4.5)$ and factor B the percentage of yeast $(0.5 \%, 1 \%, 1.5 \%)$; In the experience, the coffee was evaluated, the husk and diluted mucilage were obtained in the pulping, the $\mathrm{pH}$ was regulated and the Saccharomyces cerevisiae was added for fermentation at $20^{\circ} \mathrm{C}$, in a $10 \mathrm{~L}$ bioreactors; The physicochemical evaluation was carried out according to the analysis of variance and multiple comparisons of Tuckey $(\alpha=0.05)$ and sensory according to the Friedman test; it was determined that there is a significant difference between treatments; however, between blocks (evaluation time), there was no significant difference. It was determined that at $\mathrm{pH} 4.5$ and the addition of $3 \%$ commercial dry yeast in the fermentation of pulp and coffee husk, they allow the elaboration of an alcoholic beverage with a sensory rating between good and very good, with color values 8.15, aroma 7.82, aspect 5.87 and 7.24 flavor; also density 1.029 , $\mathrm{pH} 4.75$, total solids $2^{\circ}$ Brix, titratable acidity $8.4 \mathrm{~g} / \mathrm{L}$, alcoholic degree $15.6 \mathrm{GL}$, ethereal extract $0.193 \%$, total protein $0.42 \%$, extract free of nitrogen $0.692 \%$ and gross energy $6.911 \mathrm{kCal} / 100 \mathrm{~mL}$.
\end{abstract}

Keywords: Yeast, evaluation, fermentation, physicochemical, sensory.

\footnotetext{
Ingeniero agroindustrial, Profesor auxiliar a Tiempo completo de la Facultad de Ingeniería y Ciencias agrarias de la Universidad Nacional Toribio Rodríguez de Mendoza de Amazonas. Correo electrónico: segundo.olivares@untrm.edu.pe

${ }^{2}$ Ingeniero mecánico, Profesor asociado a Tiempo completo de la Facultad de Ingeniería y Ciencias agrarias de la Universidad Nacional Toribio Rodríguez de Mendoza deAmazonas; Correo electrónico: meregildo.silva@untrm.edu.pe

${ }^{3}$ Estudiante de la escuela profesional de Ingeniería agroindustrial; Universidad Nacional Toribio Rodríguez de Mendoza de Amazonas; Correo electrónico:7428302971@untrm.edu.pe
} 


\section{INTRODUCCIÓN}

La investigación propone una tecnología para el procesamiento de la cáscara y mucilago de café; como una alternativa consecuente para el desarrollo económico y el cuidado del medio ambiente; en el sentido que la producción de café en Perú involucra 223902 familias productoras; principalmente en regiones como Junín, San Martín y Amazonas; (Díaz \& Willems, 2017), Según la Junta nacional del café las exportaciones en el año 2019 fueron 5008183 qq; sabiendo que en promedio el $40 \%$ del café cerezo es pulpa y cáscara; se tuvo aquel año un aproximado de 2003273,2 qq de pulpa y cáscara que se habría desechado habría contaminado al medio ambiente. En la región Amazonas, los distritos de Omia con 5 668,46 ha y Lonya Grande con 5457,22 ha de cultivo de café lideran la producción (Inei, 2012).

Para desarrollar la propuesta tecnológica se sabe que la pulpa de café presenta principalmente $60,29 \%$ de extracto libre de nitrógeno en base seca, fibra $22.86 \%$ (Noriega, Silva, \& García, 2008) y el mucílago fresco tiene humedad entre $85 \%$ a $91 \%$; los azúcares son los principales componentes de su materia seca, $6,2 \%$ a $7,4 \%$, conformados por $63 \%$ de azúcares reductores, asimismo presenta levaduras y bacterias que demuestran su alta perecibilidad. (Puerta \& Ríos, 2011).

Por lo mencionado y bajo un análisis tecnológico se propuso la elaboración de una bebida alcohólica por fermentación de cáscara y mucilago del café; en consecuencia existieron varias variables que podría influenciar en la propuesta, sin embargo al revisar diversas informaciones se consideró, que la materia prima se debe cosechar frutos o cerezas maduras en horas frescas del día y no deben pasar más de ocho horas entre la recolección y el inicio de su beneficiado, que el despulpado se debe realizar con la finalidad de retirar la cascara y parte de la pulpa (mucílago). (Caballero \& Cruz, 2015).

La selección y beneficio del café es una etapa crítica ya que se menciona que la velocidad y los compuestos formados en la fermentación del café dependen de la calidad del sustrato, madurez y sanidad del fruto, (Puerta, 2012); asmismo una de las caracteristicas importantes de la cáscara y pulpa de café es la cantidad de antioxidantes equivalente a 2024,26 $\mu$ M ET/g (Serna, Torres,

Martínez, \& Hernández, 2018); lo se valora para su conservación en el proceso de fermentación.

Para el proceso de fermentación es necesario un proceso de acondicionamiento del sustrato para trabajo de la levadura S. cerivisiae; se valoró que los mayores contenidos de etanol se alcanzaron en los tratamientos: con hidrólisis enzimática con celulasas $(97,78 \%)$ y $\sin$ hidrólisis $(96,92 \%)$. (Rodríguez , 2013).

La operación de fermentación es el proceso central en la presente investigación de lo cual se menciona que con la combinación de tipos de hidrólisis e inóculos de S. cerivisiae, determinan que el mucílago sin hidrolizar e inoculado con levaduras comerciales obtuvieron un valor de $57,90 \mathrm{~mL}$ de alcohol por kilogramo de mucílago sin diluir, con $98,60 \%$ de etanol en promedio. (Rodríguez \& Zambrano, 2011).

En caso de la aptitud del sustrato para la fermentación se valoró la optimización de las condiciones para la producción de biomasa a partir de cáscara de café para el crecimiento de la levadura Candida utilis. Los resultados obtenidos sugieren que la cáscara de café constituye un sustrato adecuado para la producción de biomasa. (Carrillo, Zavala, Alvarado, Morales, \& Bautista,

2011)

La eficiencia en la producción de alcohol de la levadura S. cerevisiae se tuvo en cuanta el registro lo recomendado por de valores de $50,5 \mathrm{~g} / \mathrm{L}$ a las $24 \mathrm{~h}$, partiendo de $100 \mathrm{~g} / \mathrm{L}$ con un rendimiento del 97,2\%, en condiciones que ha permitido conocer el tiempo en el que se consume el sustrato, así como la producción de etanol. (Valdés, y otros, 2015)

Se considera que la propuesta es una innovación por lo cual se tomó como referencias para tomar cualificar la bebida obtenida productos similares de otras frutas como la elaboración de una bebida de Atemoya, usando S. cerevisiae $1.92 \%$, obteniendo un grado de alcohol de $9.77^{\circ} \mathrm{GL}$ y buena aceptación en relación a la textura, color, sabor y olor. (Martínez, Reina, Arias , Hernan, \& Agudelo, 2019); De forma similar se elaboró un vino joven de Borojó a partir del mosto fermentable, se realizaron los procesos de clarificación y filtración y se logró un contenido de

$9.25{ }^{\circ} \mathrm{GL}$ (García , Flores, \& Marrugo, 2016) y también se obtuvo una bebida fermentada de naranja, donde el mejor tratamiento se estableció presentó valores de $\mathrm{pH} 3,45$, acidez $0,39 \%$ y sólidos solubles de $6,67 \%$ con un contenido de grados alcohólicos de 13,2\%. (Remache, 2015)

De acuerdo al análisis y evaluación de la información de antecedentes e información básica se determinó que la principal dificultad para la elaboración de una bebida alcohólica a partir de mucílago y cáscara de café son los parámetros para la fermentación en relación al $\mathrm{pH}$ y la cantidad de levadura a utilizar dado que son condiciones determinantes; por lo tanto se tuvo por objetivo la determinación del valor de $\mathrm{pH}$ 
y porcentaje levadura en la fermentación.

\section{MATERIAL Y MÉTODOS}

La investigación fue de tipo experimental ya que el objetivo fue determinar el $\mathrm{pH}$ y cantidad de adición de levadura para elaborar una bebida de cáscara y mucilago de café, para tal fin se realizó el análisis fisicoquímico y sensorial del producto según cada tratamiento. Del mismo modo se trata de un estudio de laboratorio los cuales se ejecutaron en la UNTRM-A

Se utilizó un diseño de tratamientos en arreglo factorial del tipo 32 , basado en un experimento completamente al azar (Montgomery, 2004); con tres repeticiones, donde el factor $\mathrm{A}$ y el factor $\mathrm{B}$, tal como se muestra en la Tabla 1 Tabla 1. Descripción de los factores, símbolo y referencias.

\begin{tabular}{cccc}
\hline & \multicolumn{2}{c}{ Nivel del factor Factor } \\
& & Descripción & Referencia \\
& & A1 & 3.5 \\
& & A2 & 4 \\
A & & A3 & 4.5 \\
& & & \\
\hline & & B1 & $0.5 \%$ \\
B & B2 & $1 \%$ \\
& & B3 & \\
\hline
\end{tabular}

La muestra fue $10 \mathrm{~kg}$ de pulpa y cáscara de café para cada tratamiento, recolectados al azar en el proceso de despulpado de café con madurez organoléptica para su recolección.

Para la elaboración de la bebida alcohólica se realizó la recepción de la pulpa y cáscara del café sin diluir, el pesado, estandarizado del $\mathrm{pH}$ (Tratamiento), hidrolisis, se controló la fermentación (anaerobiosis, actividad de agua $(0.95-0,97)$, temperatura $\left.20^{\circ} \mathrm{C}\right)$; se adicionó la levadura S. cerevisiae (Tratamiento), en un digestor protipo, se realizó el filtrado, clarificación con bentonita; pasteurización ( 2 min a $70{ }^{\circ} \mathrm{C}$ ) y se envasó en botellas de vidrio transparente con capacidad de $750 \mathrm{~mL}$.

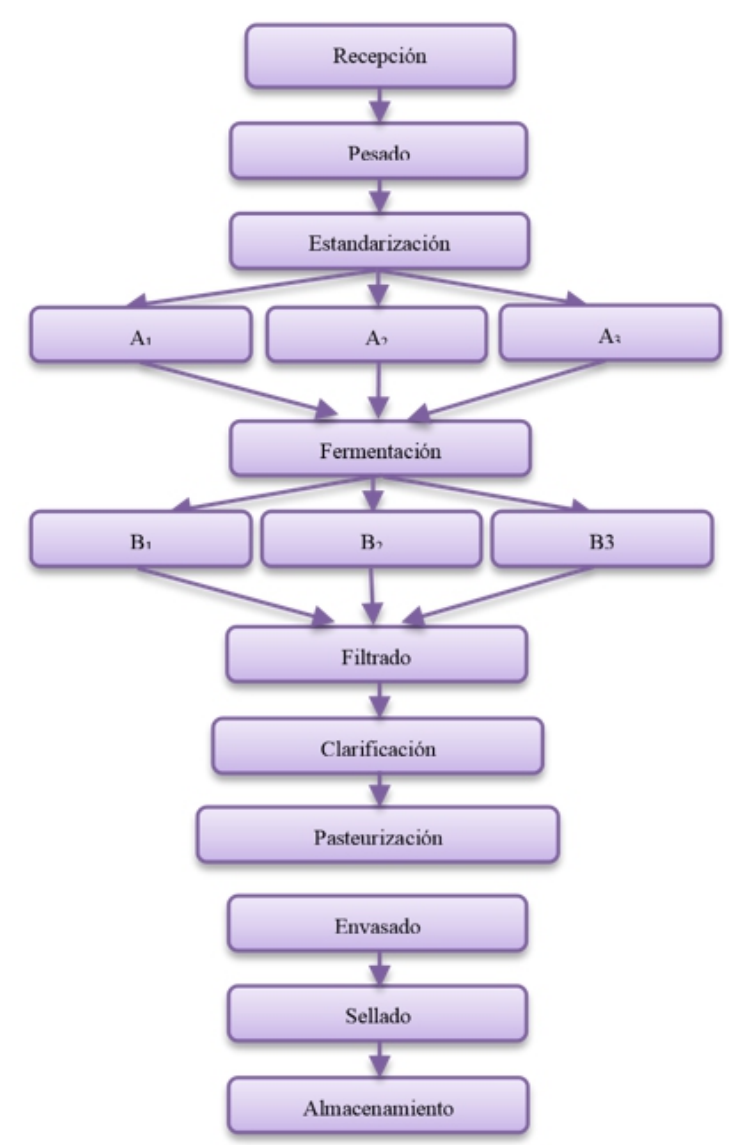

Figura 1. Flujograma para la elaboración de labebida alcohólica.

La bebida obtenida en cada tratamiento fue sometida a análisis por 4 meses, cada 30 días, el análisis sensorial con 15 panelistas no entrenados ayudados con la escala hedónica de 9 puntos de Peryamm \& Pilgrim,1957 (Hernandez, 2005) y el análisis fisicoquímico a dos mejores tratamientos; midiendo el pH (método potenciométrico (AOAC., 1990)), porcentaje de alcohol etílico (destilación fraccionada), acidez titulable (método de titulación) y proteína total $(\%)$, extracto libre de nitrógeno(\%), extracto etéreo(\%) y energía libre $(\mathrm{kCal} / \mathrm{g})$ según la AOAC. (1990).

Para el análisis de datos se empleó el software excel y Statistix 8, las características fisicoquímicas con análisis de varianza, y comparaciones múltiples de Tuckey al 95\% de confianza y el análisis sensorial mediante la prueba de Friedman. 


\section{RESULTADOS}

Características fisicoquímicas del mucílago y cáscara de café en estado fresco.

Tabla 2. Valores promedio de las características fisicoquímicas del mucilago y cáscara de café fresco

\begin{tabular}{|c|c|c|c|c|c|}
\hline Muestra & Color & Aroma & $\begin{array}{c}\text { Densidad } \\
\text { promedio } \\
\left(\mathbf{k g} / \mathbf{m}^{\mathbf{3}}\right)\end{array}$ & $\begin{array}{c}\text { Sólidos } \\
\text { totales } \\
\text { promedio } \\
\left({ }^{\circ} \text { Brix) }\right.\end{array}$ & $\begin{array}{c}\mathbf{p H} \\
\text { promedio }\end{array}$ \\
\hline $\begin{array}{c}\text { Pulpa y } \\
\text { cáscara } \\
\text { de café }\end{array}$ & $\begin{array}{c}\text { Rojo } \\
\text { intenso }\end{array}$ & Característico & 612 & 16.9 & 5.6 \\
\hline
\end{tabular}

Características sensoriales y fisicoquímicas del producto obtenido

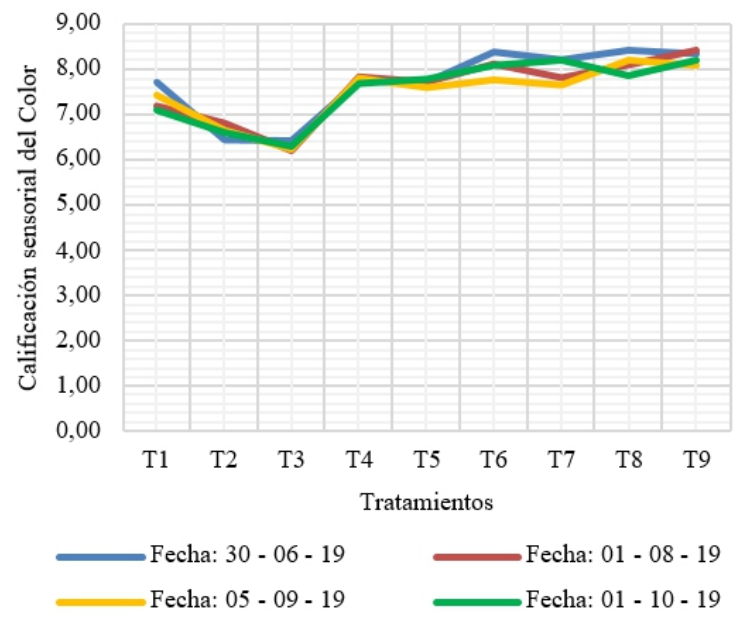

Figura 2. Calificación sensorial del color del producto elaborado, evaluado a los 30, 60, 90 y 120 días en anaquel para cada tratamiento

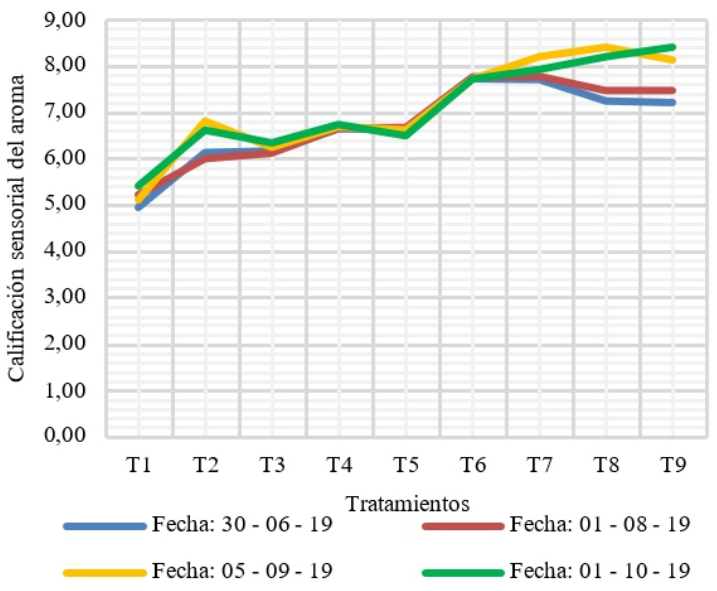

Figura 3. Calificación sensorial del aroma del producto elaborado, evaluado a los 30, 60, 90 y 120 días en anaquel para cada tratamiento

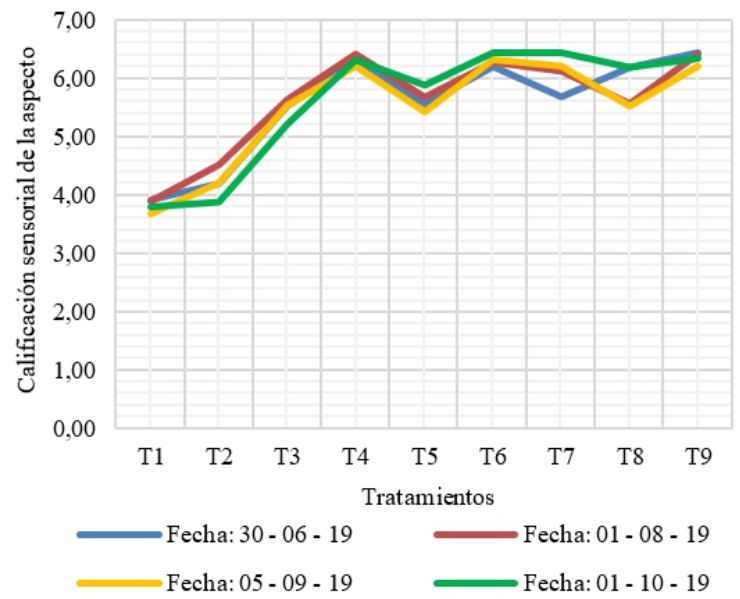

Figura 4. Calificación sensorial del aspecto del producto elaborado, evaluado a los 30,60, 90 y 120 días en anaquel para cada tratamiento.

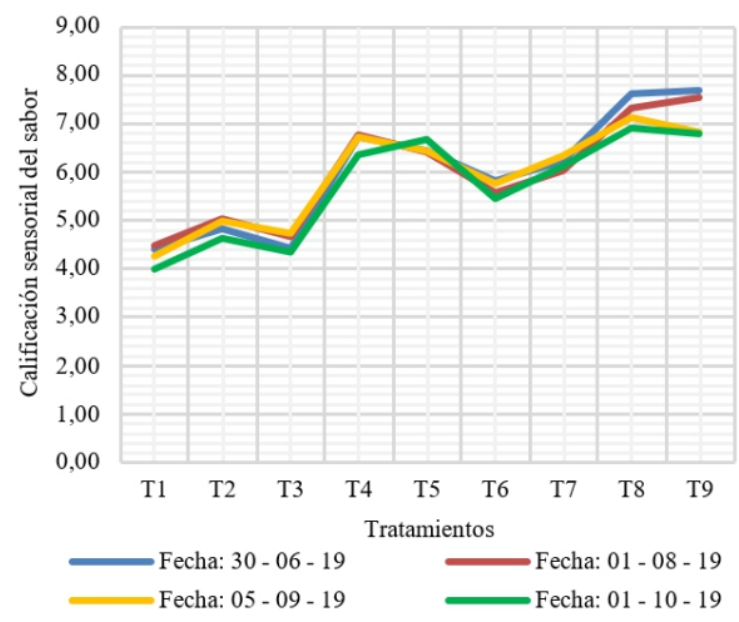

Figura 5. Calificación sensorial del sabor del producto elaborado, evaluado a los 30, 60, 90 y 120 días en anaquel para cada tratamiento.

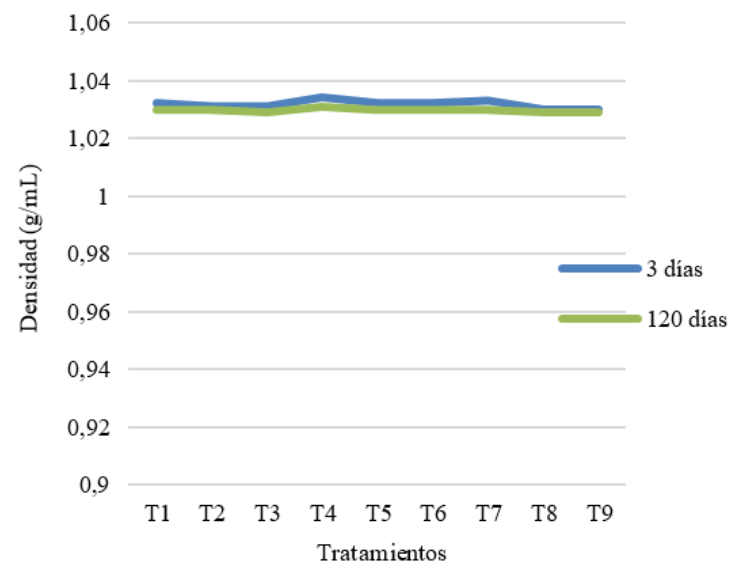

Figura 6. Densidad del producto elaborado, evaluado a los 3 y 120 días en anaquel para cada tratamiento 


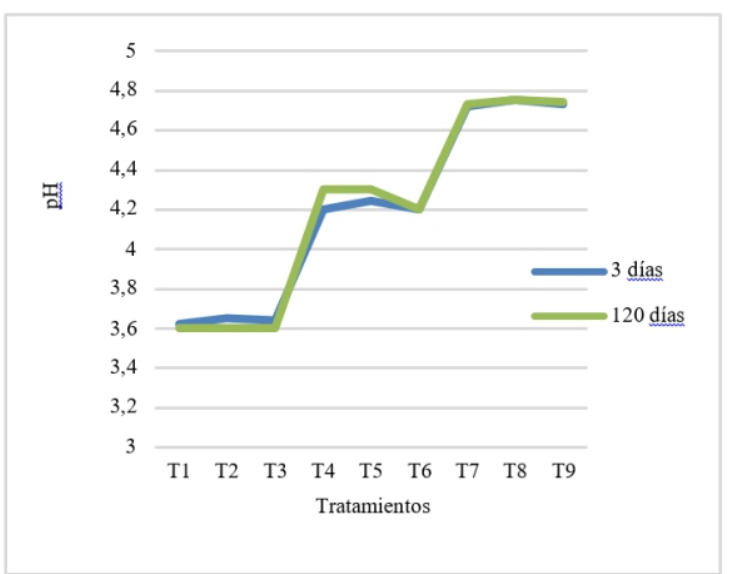

Figura 7. $\mathrm{pH}$ del producto elaborado, evaluado a los 3 y 120 días en anaquel para cada tratamiento.

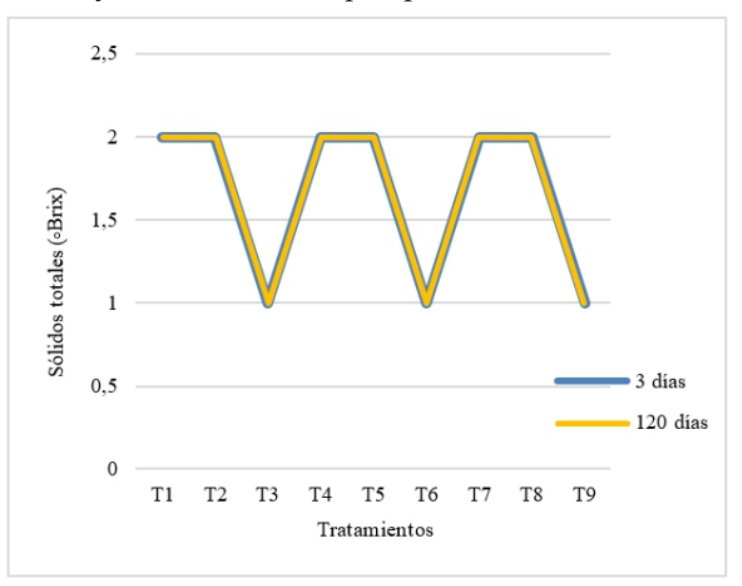

Figura 8. ${ }^{\circ}$ Brix del producto elaborado, evaluado a los 3 y 120 días en anaquel para cada tratamiento

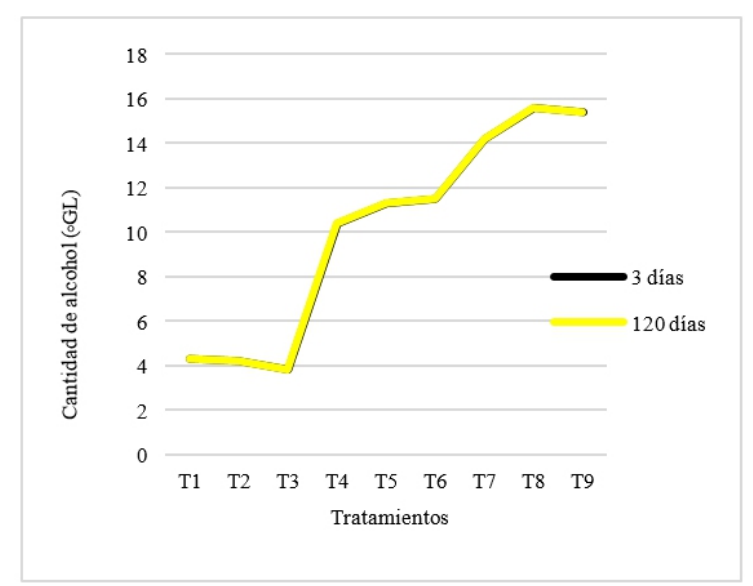

Figura 9. Cantidad de alcohol del producto elaborado evaluado a los 3 y 120 días en anaquel para cada tratamiento.

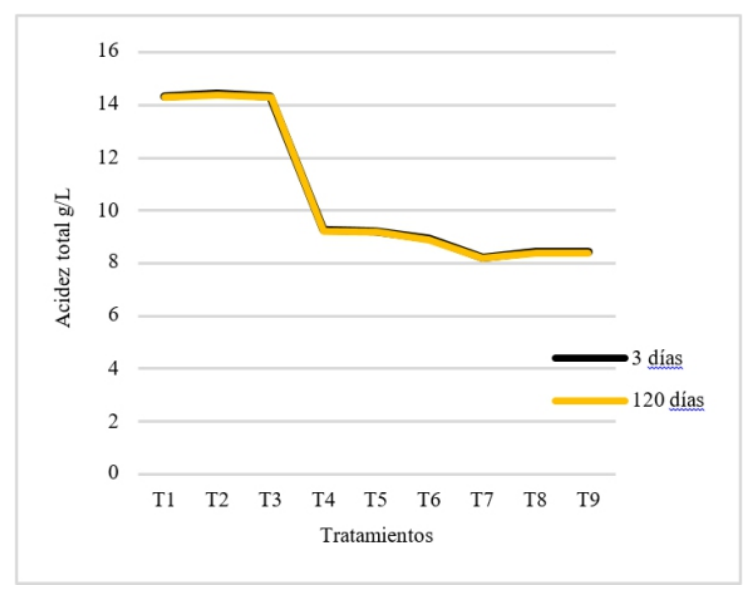

Figura 10. Acidez total del producto elaborado, evaluado a los 3 y 120 días en anaquel para cada tratamiento.

Tabla 3. Evaluación química de los mejores tratamientos

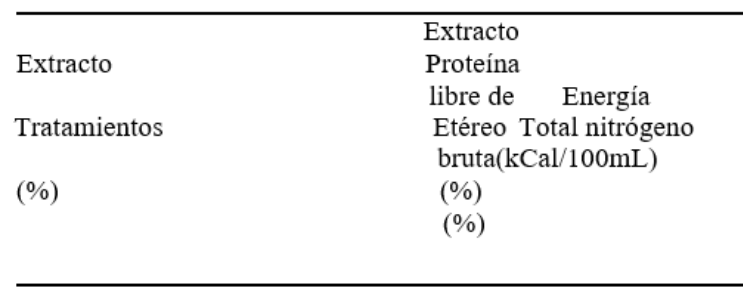

T8 0,19.3 0.42 0, 6926.911 .3 T9 0.19.4 0.44 0.683 6.9146

\section{DISCUSIÓN}

Rodríguez y Zambrano (2011), realizaron la obtención de alcohol a partir de mucilago de café; mediante combinación de tipos hidrolisis natural, ácida, alcalina y enzimática e inóculos de S. cerevisiae en forma natural, prensadas y seca comercial, resultando que el mucilago sin hidrolizar e inoculado con levadura comercial alcanzó 57,90 mL de alcohol por kilogramo de mucílago sin diluir; en la investigación efectuada también se utilizó mucilago y cáscara sin hidrolizar; además de la levadura seca comercial (S. cerevisiae); la producción de alcohol fue de $15.6^{\circ} \mathrm{GL}$ (Fig.9), para $4 \mathrm{~L}$ de bebida obtenida; aproximadamente $62.4 \mathrm{~mL}$ de alcohol por kilogramo de cáscara y mucílago; este resultado sería relacionado con los $16.6^{\circ}$ Brix de la muestra fresca y las condiciones de la fermentación principalmente en cuanto al $\mathrm{pH} 4.5$ y adición de $\mathrm{S}$. cerivisiae como levadura seca comercial en un $3 \%$.

Puerta (2012), estudió los factores de la fermentación, en particular, la velocidad y los compuestos formados en la fermentación del café demostrando que si dependen de la calidad del sustrato, madurez y sanidad del fruto; en la investigación no se evaluó la velocidad en la 
fermentación ni los compuestos formados en la fermentación, sin embargo conforme a las figura 2, 3, 4 y 5; la presencia de compuestos formados se demuestra en la calificación sensorial durante 120 días, resaltando entonces que es necesario tener especial control de los parámetros para la fermentación; principalmente el $\mathrm{pH}$ y cantidad de levadura, dando que determinan la velocidad.

Gualtieri, et al. (2007) utilizaron los residuos de la pulpa de café, rico en materia orgánica, como sustrato para la producción de biomasa de levaduras por procesos de fermentación aeróbica de $\mathrm{S}$. cerevisiae y Candida utilis, y se enriqueció con sales nutritivas; concluyéndose que la pulpa de café constituye un sustrato adecuado para obtener biomasa, en ese sentido en la investigación se utilizó la $\mathrm{S}$. cerevisiae, sin embargo no se adicionó sales nutritivas y a pesar de ello se obtuvo alta tasa de generación de alcohol obtenida en 24 horas de fermentación (Fig. 9); demostrándose así la calidad del sustrato y la eficacia de los controles en la fermentación

Cortez y Ladino (2016), en la investigación elaboración de una bebida alcohólica con residuos de café concluyen, que la fermentación inducida inoculando levaduras especializadas para la producción de vino, genera mejores características sensoriales en la fermentación que con las levaduras nativas de la pulpa o con S. cerevisiae; en la investigación se usó S. cerevisiae, obteniéndose aceptación sensorial como se observa en las figura 2, 3,4 y 5 ; sin embargo se debe mencionar que la calificación fue por panelistas no entrenados pero tienen las características de los posibles consumidores; asimismo esta aceptación se sustenta en las características que la bebida habría obtenido por el control de la fermentación.

Puerta (2015), menciona que el café cuando se fermenta a $15^{\circ} \mathrm{C}$ se incrementa la frecuencia de sabores especiales y de café con calidad especial y superior, en comparación a la calidad obtenida a temperatura ambiente de 18 a $26^{\circ} \mathrm{C}$; en la investigación se realizó por su puesto la fermentación solo de cáscara y mucilago a una temperatura promedio de $20^{\circ} \mathrm{C}$, sin embargo se obtuvo calificación sensorial aceptable la que no fue realizada por un catador experto; pero es válida para los proyectos de comercialización del producto.

Arguedas (2014), efectuó pruebas de producto en desarrollo para definir la preferencia del consumidor de una bebida fermentada de pulpa de café, utilizando cinco cepas diferentes de levaduras: S. Cerevisiae Bayanus S6U, S.C. Davis 522, S.C. Levuline ALS (levadura seca activa) y S.C. Vitilevure C y SC comercial como levadura de panificación y no encontró diferencias significativas en la aceptación por parte del consumidor; basado en ello en la investigación se utilizó S. Cerevisiae comercial como levadura de panificación pero se tuvo especial cuidado en la fermentación el control de $\mathrm{pH}$ y cantidad de adición de levaduran como variables de estudio; se infiere que de ello dependió la performance de las buenas caracteristicas sensoriales y fisicoquímicas observadas en los resultados

García , Flores, \& Marrugo (2016), elaboraron un vino joven de borojó a partir del mosto fermentable de la caracterización fisicoquímica se sabe que presentó un contenido de $9.25^{\circ} \mathrm{GL}$, lo cual menciona que cumple la Norma Técnica

Colombiana 708 para bebidas alcohólicas vinos de frutas; en la investigación se elaboró una bebida fermentada de cáscara y mucilago de café, alcanzó $15.6^{\circ} \mathrm{GL}$, que es un valor que estaría dentro de los niveles permitidos para el consumo; sin embargo al ser un producto nuevo aún se necesitaría una norma específica.

\section{CONCLUSIONES}

Se determinó que a pH 4.5 y adición de levadura en $3 \%$ en la fermentación de pulpa y cáscara de café (Tratamiento 8), permiten la elaboración de una bebida alcohólica con características fisicoquímicas y sensoriales para el consumo.

Las características fisicoquímicas y sensoriales de la bebida obtenida según el mejor tratamiento registra variación estadísticamente significativa durante los 120 días de evaluación, manteniéndose en valores promedio de color 8.15 , aroma 7.82 , aspecto $5.87 \mathrm{y}$ sabor 7.24; asimismo densidad 1.029, $\mathrm{pH} 4.75$, solidos totales $2^{\circ}$ Brix, acidez titulable $8.4 \mathrm{~g} / \mathrm{L}$, grado alcohólico $15.6 \mathrm{GL}$, extracto etéreo $0,193 \%$, proteína total $0.42 \%$, Extracto libre de nitrógeno 0,692\% y Energía bruta $6.911 \mathrm{kCal} / 100 \mathrm{~mL}$.

La vida útil de la bebida alcohólica a partir de la fermentación de mucílago y cáscara de café mínimo es 120 días durante las cuales la bebida ha demostrado un proceso de baja variabilidad en sus características fisicoquímicas y sensoriales.

\section{REFERENCIAS BIBLIOGRAFICAS}

AOAC. (1990). Association of Official Analytical Chemists. Official Methods of Analysis of the.AOAC. Washington, USA. 15 th edition.

Arguedas, P. (2014). Procedure definition to obtain a fermented drink from the coffee pulp.

Costa Rica, 38-49. Retrieved f r o m http://revistas.tec.ac.cr/index.php/tec_march a/article/view/1654 
Carrillo, M. L., Zavala, D., Alvarado, B., Morales, K. S., \& Bautista, P. (2011). Obtención de biomasa a partir de cáscara de café. Revista académica científica Tlatemoani, 14.

Cortés, M. E., \& Ladino, O. B. (2016). Elaboración de una bebida alcohólica usando subproductos del proceso de beneficio del café (Pulpa de café). Revista Nova (Colombia), 1-6.

Díaz, C., \& Willems, M. C. (2017). Línea de Base del sector café ene el Perú. Lima: Programa de las Naciones Unidas para el Desarrollo PNUD. Retrieved $\mathrm{f} \quad \mathrm{r} \quad \mathrm{o} \quad \mathrm{m}$ http://www.minagri.gob.pe/portal/downloa d/ 2017/pncafe/sector-cafe-peru.pdf García , L.

A., Flores, C. I., \& Marrugo, Y. (2016). Elaboración y caracterización fisicoquímica de un vino joven de fruta de borojó (B patinoi Cuatrec). Ciencia, Docencia y Tecnología, 507-519.

Gualtieri, M. J., Villalta, C., Díaz, L. E., Medina, G., Lapenna, E., \& Rondón, M. E. (2007). Producción de biomasa de Saccharomyces cerevisiae y Candida utilis usando residuos de pulpa de Coffea arabica L. Revista del Instituto Nacional de Higiene Rafael Rangel, 31-37. Retrieved from Disponible en $<\mathrm{http}$ ://ve.scielo.org/scielo.php?script $=\mathrm{sci}$ a r t t e x t \& p i d = S 0798 04772007000200004\&lng=es\&nrm=iso>

Hernandez, E. (2005). Evaluación sensorial. Bogotá: Facultad de ciencias básicas e ingenniería - Universidad nacional abiertay adistancia.

Inei. (2012). Censo nacional agrario. Lima - Perú: Instituto nacional de estadistica e informática. Martínez, J., Reina, B., Arias , A. L., Hernan, J. C., \& Agudelo, J. H. (2019). Caracterización fisicoquímica, microbiológica y sensorial del licor obtenido a partir del fruto de atemoya (Annona cherimola $\times$ Annona squamosa). Scientia et Technica Año XXIV, 636-642. doi: http://dx.doi.org/10.22517/23447214.21501

Montgomery, D. C. (2004). Diseño y análisis de experimentos . México: Limusa S.A. Wiley.

Noriega, A., Silva, R., \& García, M. (2008). Utilización de la pulpa de café en la alimentación animal. Zootecnia tropical, 411- 419 .

Puerta, G. I. (2012). Factores, procesos y controles en la fermentación del café . Chinchiná : Cenicafé (Avances Técnicos No. 422), 12.

Puerta, G. I. (2015). Fermentación controlada del café: Tecnología para agregar valor a la calidad. Avances técnicos Cenicafé, 12.

Puerta, G. I., \& Ríos, S. (2011). Composición química del mucilago de café según el tiempo de fermentación y refrigeración. Cenicafé, 2(62), 23-40.

Remache, H. E. (2015). Obtención de una bebida fermentada de naranja (Citrus sinensis) aplicando la enzima Peptinasa (PEC-600) como clarificante. Los Ríos - Ecuador: Universidad técnica estatal de Quevedo Escuela de Ingeniería para el desarrollo agroindustrial.

Rodríguez, N. (2013). Producción de alcohol a partir de la pulpa de café. Centro Nacional de Investigaciones de Café, Cenicafé, 78-93.

Rodríguez, N., \& Zambrano, D. A. (2011). Producción de alcohol a partir de mucílago de café. Centro Nacional de Investigaciones de Café, Cenicafé, 59 - 69.

Serna, J. A., Torres, L. S., Martínez, K., \& Hernández, M. C. (2018). Aprovechamiento de la pulpa de café como alternativa de valorización de subproductos. Revista Ion, 37-42.

Valdés, A., Fernandes, B. D., Mota, A. M., Aguilar, C. N., Llina, A., Teixeira, J. A., \& Ruiz, H. A. (2015). Cinética para la producción de bioetanol usando levadura Saccharomyces cerevisiae PE-2 para su escalamiento en reactores en columna y gas-

LIFT. XVI Congreso $\mathrm{N}$ a c i o n a 1 de Biotecnología y Bioingeniería, 2. 